University of Nebraska - Lincoln

DigitalCommons@University of Nebraska - Lincoln

Publications, Agencies and Staff of the U.S.

Department of Commerce

U.S. Department of Commerce

$9-2006$

\title{
Genetic monitoring as a promising tool for conservation and management
}

Michael K. Schwartz

USDA Forest Service, mkschwartz@fs.fed.us

Gordon Luikart

University of Montana, gordon.luikart@umontana.edu

Robin Waples

NOAA, robin.waples@noaa.gov

Follow this and additional works at: https://digitalcommons.unl.edu/usdeptcommercepub

Schwartz, Michael K.; Luikart, Gordon; and Waples, Robin, "Genetic monitoring as a promising tool for conservation and management" (2006). Publications, Agencies and Staff of the U.S. Department of Commerce. 482.

https://digitalcommons.unl.edu/usdeptcommercepub/482

This Article is brought to you for free and open access by the U.S. Department of Commerce at DigitalCommons@University of Nebraska - Lincoln. It has been accepted for inclusion in Publications, Agencies and Staff of the U.S. Department of Commerce by an authorized administrator of DigitalCommons@University of Nebraska - Lincoln. 


\title{
Genetic monitoring as a promising tool for conservation and management
}

\author{
Michael K. Schwartz ${ }^{1}$, Gordon Luikart ${ }^{2,3}$ and Robin S. Waples ${ }^{4}$ \\ ${ }^{1}$ USDA Forest Service, Rocky Mountain Research Station, 800 E. Beckwith Avenue, Missoula, MT 59801, USA \\ ${ }^{2}$ Center for Investigation of Biodiversity and Genetic Resources, University of Porto, Campus Agràrio de Vairão 4485-661, Portugal \\ ${ }^{3}$ Division of Biological Sciences, University of Montana, Missoula, MT 59812, USA \\ ${ }^{4}$ National Marine Fisheries Service, Northwest Fisheries Science Center, 2725 Montlake Boulevard East, Seattle, WA 98112, USA
}

In response to ever-increasing anthropogenic changes to natural ecosystems, regional, national and international organizations have established guidelines for monitoring biological diversity. Most monitoring programs, however, do not take full advantage of the potential afforded by molecular genetic markers, which can provide information relevant to both ecological and evolutionary time frames, while costing less and being more sensitive and reliable than traditional monitoring approaches. As several molecular and computational approaches are relatively new, many technical and theoretical issues remain to be resolved. Here, we illustrate how DNA and population genetic data can provide valuable information, often unattainable via other approaches, for monitoring species of management, conservation and ecological interest.

\section{Introduction}

It is becoming increasingly important to monitor unintended consequences of anthropogenic changes on natural populations. Although many national and international organizations have established principles and strategies for monitoring biological diversity [1-3], little use is made of the benefits afforded by molecular genetic markers. Meanwhile, new laboratory and statistical techniques now enable the use of molecular markers for genetic monitoring of wild populations [4-7]. Although the term 'genetic monitoring' is becoming widely used throughout the ecological, evolutionary, wildlife and conservation fields, there is no consensus, and some confusion, regarding its definition. To some, genetic monitoring is simply the use of genetic data to study demography or more complex evolutionary and ecological processes, whereas to others it implies systematic measurements of population genetic parameters over time.

Here, we define genetic monitoring as quantifying temporal changes in population genetic metrics or other population data generated using molecular markers. We distinguish monitoring, which must have a temporal dimension, from assessment, which reflects a snapshot of population characteristics at a single point in time. For instance, the term 'molecular genetic monitoring' has been used to refer to estimates of the proportion of whales from protected stocks that appeared in Asian

Corresponding author: Schwartz, M.K. (mkschwartz@fs.fed.us).

Available online 7 September 2006. markets [8]. We consider this an assessment because data from a seven-year period were pooled to provide a single point-estimate. A monitoring study of whale-meat markets would evaluate whether the fraction of protected species changes over time.

We separate genetic monitoring into two categories: Category I includes the use of diagnostic molecular markers for traditional population monitoring through the identification of individuals, populations, and species, whereas Category II includes the use of genetic markers to monitor population genetic parameters (Figure 1).

\section{Category I. Diagnostic molecular markers for traditional population monitoring}

Diagnostic assays that use molecular markers can identify individuals, populations, species and other taxonomic levels. We distinguish methods using molecular markers to identify individuals (Figure 1, Category Ia) from those identifying species or other groups (genera, subspecies or populations; Category Ib).

\section{Category la. Identifying individuals}

Molecular tags can be used in lieu of physical tags or natural markings to identify individuals. Data generated from such tags are often used in traditional population ecology models estimating abundance or vital rates. Below we describe several metrics commonly monitored in wild populations that have profited from DNA-based methods.

Abundance The most common metric used for monitoring animal populations is abundance (Box 1). With rare species, abundance indices, such as the number of animals detected, have proven relatively inexpensive to obtain through non-invasive genetic sampling. DNA-based abundance indices are often adjusted to remove biases associated with limited sample size or imperfect detection rates. These adjustments can be accomplished by applying asymptotic analyses or using models that account for covariates associated with capture, such as season, time of day, or habitat $[9,10]$.

Abundance can also be estimated through capture-mark-recapture (CMR) analyses (see Glossary). Traditionally, this has required physical capture and marking of a sample, followed by release and subsequent recapture of a proportion of marked animals (reviewed in Ref. [11]). Because each individual has a unique, naturally occurring genetic code (typically revealed with 


\section{Glossary}

Admixture: composite gene pool of $F_{1}, F_{2}, \ldots F_{x}$ individuals resulting from hybridization between two or more populations.

Approximate Bayesian computation (ABC): a statistical framework using simulation modeling to approximate the Bayesian posterior distribution of parameters of interest (e.g. $N_{e}, N m$ ), often by using multiple summary statistics (e.g. $H_{e}, A, F_{S T}$ ). It is computationally quicker than full Bayesian approaches, with minimal loss of accuracy and precision.

Assignment method: any of several related statistical methods that use genetic information to ascertain population membership of individuals.

Barcoding: the use of short, standardized DNA sequences, typically from a mitochondrial gene, to identify and discover species quickly and easily.

Capture-mark-recapture (CMR): field sampling method used to estimate population size or vital rates (i.e. survival, recruitment, movement).

Effective population size $\left(\boldsymbol{N}_{e}\right)$ : the size of an ideal population that would have the same rate of genetic change as the population under consideration. $N_{e}$ influences the rate of loss of genetic variation, the efficiency of natural selection and the accumulation of mutations. As a rough guideline, $N_{e}$ approximates the number of breeding individuals producing offspring that live to reproductive age.

$\boldsymbol{F}_{\mathrm{ST}}$ : standardized index of the distribution of genetic variation between populations on a scale between 0 (identical allele frequencies among populations) and 1 (populations fixed for different alleles).

$\boldsymbol{F}_{\text {temporal: }}$ a standardized measure of change in allele frequency between two or more samples collected at different times from the same population. $F_{\text {temporal }}$ is commonly used to estimate $N_{e}$.

Gametic (linkage) disequilibrium (LD): non-random association of alleles at two or more loci.

Genome typing: simultaneous genotyping of tens or hundreds of loci from across the genome, which ideally includes mapped loci and different classes of loci, such as neutral and candidate adaptive loci.

Mixture: group of $F_{0}$ individuals originating in different populations.

Non-invasive genetic sampling: collecting biological samples, such as hair, feces, or urine, from an animal without having to physically restrain, capture, or even see the animal.

Open mark-recapture model: an open population model assumes that the population can change through processes such as births, deaths, emigration and immigration during the course of the study, whereas closed population models assume the population does not change in such ways while under investigation.

Retrospective monitoring: monitoring changes from historical conditions by examining DNA extracted from previously archived samples.

microsatellites), non-invasive genetic sampling of hair, scat, feathers or other materials can be used to identify individuals, obviating the need to capture and mark individuals physically [12]. Consequently, non-invasive CMR analysis is usually used on species that are rare, difficult, dangerous, or expensive to capture (e.g. Box 1; [13,14]). For example, in Australia, microsatellite DNA and CMR analysis has provided a feasible and cost-effective strategy for monitoring long-term changes in brush-tailed rock-wallaby Petrogale penicillata abundance [15].

Vital rates Vital rates, such as survival and recruitment, drive population change over time and are of fundamental importance to population ecologists. Traditionally, survival has been difficult to estimate, much less monitor, for rare and elusive species. The ability to use molecular data to track known individuals can either replace or augment traditional efforts to monitor survival [16]. For instance, naturally shed feathers of Eastern imperial eagles Aquila heliaca have been used in a microsatellite analysis to identify individuals, monitor population turnover and estimate annual survivorship over a four-year period [17]. The results showed that turnover rates varied annually and that cumulative survival was lower than expected; such information can not be obtained easily using traditional approaches.

We expect that genetic monitoring of vital rates will become prevalent in reintroduction efforts, where post-release genetic monitoring is badly needed, but currently underused. This approach will be most compelling when genotypes of reintroduced animals can be determined before release [18] and the fate of each individual tracked by the presence or absence of its genotype in subsequent, non-invasive samples. These data can also be used for parentage analysis to monitor reproductive output.

\section{Category Ib. Identifying species and other groups}

Diagnostic genetic markers can be used to identify species or other groups (e.g. genetically differentiated populations, subspecies, or genera) in addition to individuals. The combination of non-invasive genetic sampling and novel species identification tools enables the monitoring of (i) changes in site occupancy or the geographical range of a taxon; (ii) the presence of hybrids; and (iii) the emergence of disease or invasive species (Figure 1). This category differs from similar approaches covered in Category II, where gene frequencies and assignment methods are used to assign individuals statistically to populations or species; here molecular markers are diagnostic for the taxon of interest, and probabilistic analyses based on gene frequencies are not required.

Site occupancy and geographical range Changes in the proportion and distribution of sites where a species is observed is commonly monitored to elucidate effects of environmental change $[19,20]$. These occupancy statistics can be hindered by low probability of detection owing to the elusive nature of some species, and by false positives that occur when visual identification is cryptic or a species leaves signs that are easily confused with signs of other species. Because species identification through DNA is reliable and assays are inexpensive, we expect that the use of non-invasive collection devices will soon form the basis of many large-scale surveys monitoring changes in occupancy and geographical range. Several large-scale, DNA-based surveys have already been established to monitor the distribution of species over time [21,22]. For example, the presence of Canada lynx Lynx canadensis has traditionally been monitored using unreliable metrics, such as pelt returns, snowtracks (which have high false positive rate) and incidental sightings. In 1998, a five-year monitoring effort was initiated using non-invasive genetic sampling for lynx presence on $\sim 44$ million ha of US national forest land [21,23]. Sampling was conducted using bait stations that attracted lynx to the site and elicited a rubbing response on a hair collection device. Lynx were detected in multiple locations from the hair samples using mtDNA. The success of this work provides a baseline to monitor long-term changes in the geographical range of the lynx and can already be compared with historical records [21].

Hybridization Regular monitoring with molecular markers can provide early detection of hybridization, thus giving managers a wider range of options than is available if introgression is already extensive before detection. In recognition of this reality, genetic monitoring of hybridization has been proposed for many species, including Canada lynx-bobcat Lynx rufus [24], coyote Canis latrans-red wolf Canis rufus [25], and spotted owl Strix occidentalis-barred 


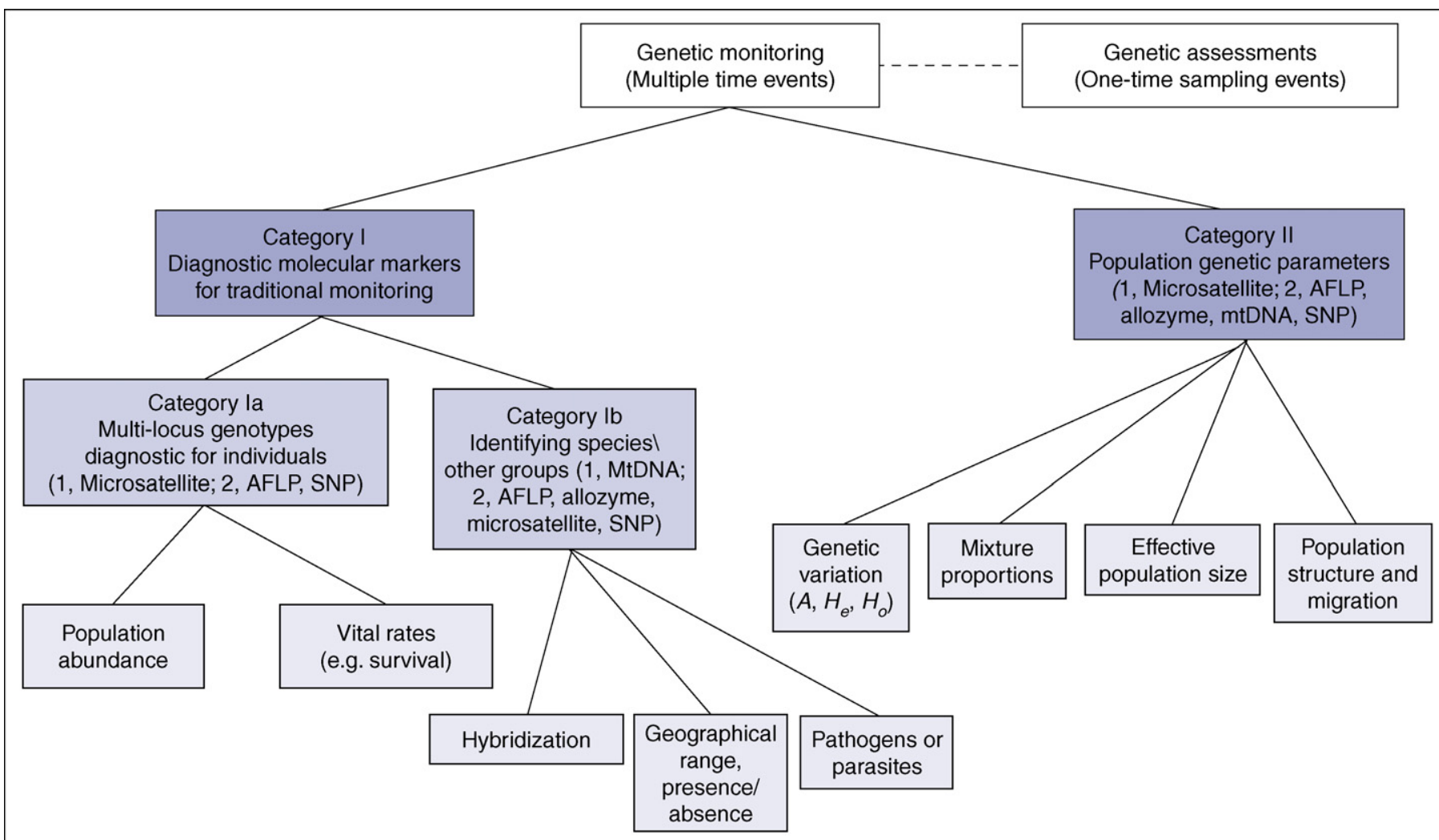

$\overline{T R E N D S ~ i n ~ E c o l o g y ~ \& ~ E v o l u t i o n ~}$

Figure 1. A framework for categorizing genetic monitoring efforts. The categories are guidelines to help direct discussions and spur research on genetic monitoring, rather than being necessarily mutually exclusive. Category I is monitoring traditional parameters using diagnostic molecular markers as tags to identify either individuals (la), or species or other groups (lb). Category II is the monitoring of population genetic parameters (e.g. $A, H_{e}, N_{e}, N m, F_{\mathrm{ST}}$; defined in Table 1 ) over time. For each category, the most commonly used molecular markers (designated 1,) and other markers widely used (designated 2,) are listed.

owl Strix varia hybridizations [26]. Genetic monitoring programs have already been initiated for several species; for example, native westslope cutthroat trout Oncorhynchus clarki lewisi in the Rocky Mountains are threatened with genomic extinction owing to widespread hybridization with introduced rainbow trout $O$. mykiss [27,28]. Diagnostic molecular assays applied to trout from the Flathead River system of Northwest Montana have demonstrated an increase in the proportion of streams with westslope cutthroat-rainbow trout hybrids between 1984 and 2001 [27].

Pathogens and parasites Disease is one of the greatest threats to population persistence. Molecular genetic markers, including DNA barcoding markers [7], have enormous potential to help monitor the presence, prevalence and transmission of pathogenic organisms. Whole genomes and gene sequences are available for increasing numbers of pathogens, making marker development relatively easy. For example, brucellosis and TB, both caused by bacterial pathogens, are among the most problematic diseases in ungulates and some carnivores [29,30]; they cost millions of dollars through vaccinations, 'test-and-slaughter programs' and reduced numbers of economically valuable wildlife and livestock. Genomes were recently sequenced for both pathogens (Brucella abortus and Mycobacterium tuberculosis, respectively), facilitating development of molecular markers to diagnose the presence of different strains and to track their spread [31].
Similarly, genetic monitoring provides new insights into West Nile virus (WNV) epidemics in humans. Over a five-month period, molecular markers were used to detect the presence of WNV-carrying Culex pipiens mosquitoes in the Washington $\mathrm{DC}$ and Baltimore, MD area of the USA; whether the mosquito was engorged with avian or mammalian blood; and the presence of WNV RNA infection in the mosquito. Temporal patterns in the molecular results, combined with temporal abundance data for mosquitoes and birds, suggests that the increase in human WNV infections during late summer is due to mosquitoes shifting their diet from their preferred avian host to humans [32].

\section{Category II. Monitoring population genetic parameters} Monitoring population genetic metrics (Figure 1) can provide insights into demographic and evolutionary processes in natural and captive (Box 2) populations that are difficult or impossible to obtain using traditional methods (e.g. temporal dynamics of a seed bank [33]). This type of monitoring can evaluate population characteristics (e.g. effective population size, $N_{e}$, or connectivity) before a monitoring program begins, as technical advances provide increasingly reliable DNA recovery from archived material (e.g. museum skins, fish scales or trophy collections). This enables 'retrospective monitoring' to assess historical conditions $[34,35]$. Here, we discuss the most commonly monitored population genetic signals. 


\section{Box 1. Monitoring for trend in abundance: Owikeno Lake} grizzly bears

As genetic monitoring is a relatively recent phenomenon, most studies using molecular tags to generate trends in abundance have asked whether the population has changed in size over only one or two time intervals. However, long-term data sets with multiple abundance estimates are generally required to distinguish meaningful changes in abundance from sampling error and environmental variability $[64,70]$. One of the best examples of estimating trends using molecular markers and Category I monitoring is the study by Boulanger et al. [14] on the influence of local salmon availability on the trend in grizzly bear Ursus arctos horribilis abundance in the Owikeno Lake area of British Columbia, Canada.

Traditionally, estimating bear abundance trend was limited by the short sampling seasons, cost, the inability to capture and follow or recapture sufficient numbers of animals, and the risk in conducting such work [71]. Boulanger et al. used DNA obtained from hair snares to mark and track individual bears [13] (Figure I), while simultaneously using traditional methods to monitor salmon availability in multiple parts of their study area. Although using DNA-based CMR to estimate single year abundance is becoming commonplace with bears $[13,72]$, Boulanger et al.'s approach was unique in that they had a five-year data set and could apply an open mark-recapture model to the entire data set [73] to estimate apparent survival, rates of recapture, additions (immigration and births) and population size changes. The authors found that, in all three sampling areas, bears had significant negative population growth in the first two years of the study, followed by positive growth in the next two. The combined experimental design enabled Boulanger et al. to elucidate the underlying mechanisms behind this trend; apparent survival and rates of addition were both related to salmon availability.

This study demonstrates that DNA-based abundance estimates can be computed in a formal modeling approach over time to make inferences regarding trends, the basis for a genetic monitoring approach.

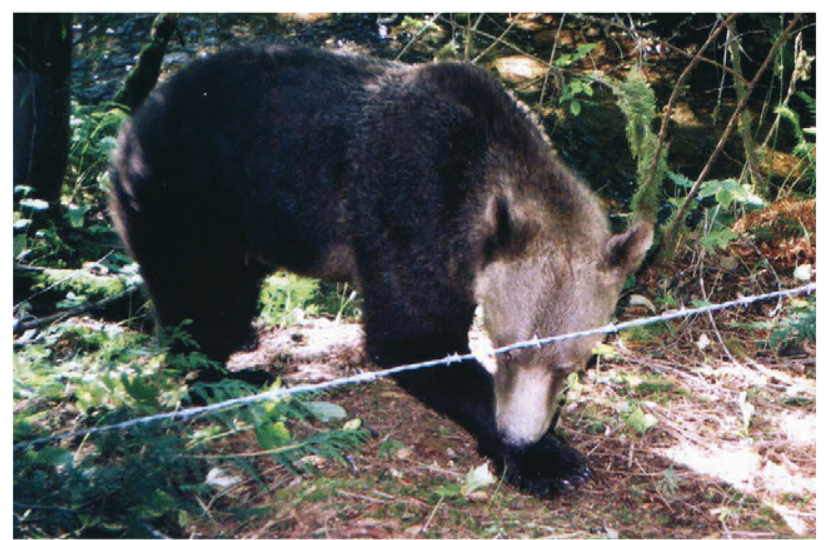

Figure I.

Population size Population genetic monitoring often evaluates changes in abundance inferred through changes in genetic diversity (e.g. expected heterozygosity, $H_{e}$; allelic diversity, $A$ ), allele frequencies (e.g. temporal changes in allele frequency, $F_{\text {temporal }}$ ) or $N_{e}$ (Table 1). Empirical studies $[36,37]$ and simulations $[38,39]$ both demonstrate that $A$ is more sensitive than is $H_{e}$ for detecting a reduction in population size, because $H_{e}$ is insensitive to the loss of rare alleles. Consequently, $A$ is often the target of monitoring efforts. Monitoring $F_{\text {temporal }}$ is an even more sensitive indicator of population decline than is loss of $A$ [37], because changes in allele frequency can be substantial without any loss of alleles [38]. For example, studies of brown trout Salmo trutta in Demark have monitored

\section{Box 2. Genetic monitoring of captive populations}

Although we focus here on monitoring wild populations, artificial propagation is being used increasingly as a conservation and management tool. Many captive programs release individuals into the wild, and informed management requires monitoring of the genetic consequences of these releases on natural populations. Here, we discuss two ways in which such monitoring can assist recovery of wild populations.

\section{Monitoring changes within captive populations}

It is important to monitor genetic changes within captive populations, as the loss of genetic variation from the use of too few founders or faulty breeding protocols can compromise recovery efforts [74]. Dowling et al. [75] monitored gene frequencies for seven years in samples of repatriated (wild-produced larvae reared in captivity and re-released as juveniles) razorback sucker Xyrauchen texanus in Arizona to determine whether the captive-rearing program was transmitting sufficient genetic variation to the endangered wild population. Their results suggest that, to date, the program has been successful in avoiding use of progeny from only a small fraction of wild spawners. By contrast, monitoring of the hatchery population of the endangered Rio Grande silvery minnow Hybognathus amarus between 2001 and 2003 has consistently shown heterozygosity levels equal to, but allelic diversity much lower than, that of the wild population [76], a classic sign of a population bottleneck.

\section{Monitoring recruitment in wild populations}

Genetic monitoring can be an efficient way of determining whether captive individuals are recruited into wild populations [77], and it is the most reliable method to determine whether they are making a reproductive contribution to subsequent generations. Hansen [78] examined long-term impacts of intense stocking on brown trout Salmo trutta in two Danish populations by comparing genetic profiles from contemporary samples with those from archived fish scales; one population changed dramatically over time owing to stocking, whereas the other showed little genetic contribution of stocked fish.

Molecular markers can also be used in parentage analysis to assess reproductive success of captive individuals in the wild. Araki et al. [79] monitored a supplementation program for steelhead (anadromous Oncorhynchus mykiss; Figure I) in Oregon and found that the relative reproductive success of hatchery fish improved when the program phased out the non-native hatchery stock and began using local native fish for brood stock. Monitoring the genetic contribution of captive individuals to natural populations is necessary before one can assess how the captive program affects the $N_{e}$ of the combined captive-wild system $[50,80]$.

Genetic monitoring of captive propagation is common in fishes, especially salmonids [61], but is increasingly being applied to other taxa. Recent studies include evaluations of the effects of oysters transplanted from Louisiana on local Chesapeake Bay populations; spread of ectomycorrhizal fungi used to enhance forest tree growth after out-planting; diversity in translocated populations of wallabies in Australia; and the success of protocols to introduce an endangered annual plant to restored vernal pools in southern California [81-84].

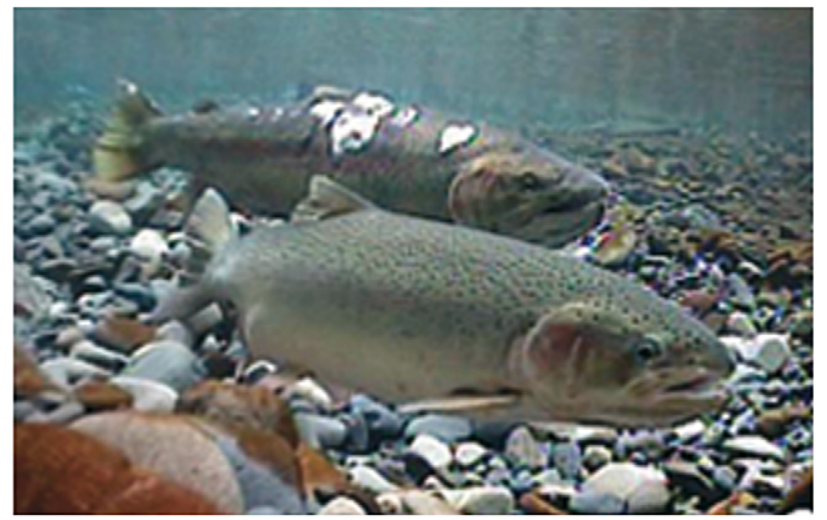

Figure $\mathrm{I}$. 
Table 1. Population genetic parameters and statistical tests for detecting genetic change resulting from a change in population size $\mathbf{e}^{\mathrm{a}, \mathrm{b}}$

\begin{tabular}{|c|c|c|c|c|}
\hline $\begin{array}{l}\text { Population genetic } \\
\text { parameters }\end{array}$ & Statistical tests for genetic change & Limitations & Advantages & Refs \\
\hline$H_{e}$ & $\begin{array}{l}\text { Paired } t \text { test, Wilcoxon's } \\
\text { signed rank test }\end{array}$ & Low power & $\begin{array}{l}\text { Provides a measure of } \\
\text { individual inbreeding } \\
\text { and is well studied } \\
\text { and widely used }\end{array}$ & {$[36,38]$} \\
\hline$A$ & $\begin{array}{l}\text { Paired } t \text { test, Wilcoxon's } \\
\text { signed rank test }\end{array}$ & $\begin{array}{l}\text { Power depends on number and } \\
\text { frequency of alleles }\end{array}$ & Potentially high power & {$[36,38]$} \\
\hline$F_{\text {temporal }}$ & $\begin{array}{l}\text { Chi square statistic, bootstrap re- } \\
\text { sampling loci to test for } F>0.0\end{array}$ & Power depends on number of alleles & High power & {$[37,38,66]$} \\
\hline $\begin{array}{l}\text { Allele frequency } \\
\text { distribution }^{c}\end{array}$ & $\begin{array}{l}\text { Kolmogorov-Smirnov test for } \\
\text { difference (change) in shape }\end{array}$ & $\begin{array}{l}\text { Power depends on allele frequency } \\
\text { distribution }\end{array}$ & Moderate-low power & [38] \\
\hline$N_{e}$ based on LD & $\begin{array}{l}\text { Compare Cls for different } \\
\text { estimates of } N_{e}\end{array}$ & $\begin{array}{l}\text { Limited evaluations; LD can be caused } \\
\text { by factors other than population size }\end{array}$ & $\begin{array}{l}\text { Power increases rapidly } \\
\text { with number of loci } \\
\text { assayed }\end{array}$ & {$[43,45,46]$} \\
\hline$N_{e}$ based on $F_{\text {temporal }}$ & $\begin{array}{l}\text { Compare Cls for different } \\
\text { estimates of } N_{e}\end{array}$ & Power depends on number of alleles & High power & {$[42,67-69]$} \\
\hline $\begin{array}{l}N_{e} \text { based on multiple } \\
\text { summary statistics } \\
\left(F_{\text {temporal }}, F_{\mathrm{IS}}, H_{e} \text { LD) }\right.\end{array}$ & $\begin{array}{l}\text { Compare Cls for different } \\
\text { estimates of } N_{e}\end{array}$ & Limited evaluations & High power & [69] \\
\hline
\end{tabular}

$H_{e}, A$ and $F_{\text {temporal }}$ for five time periods from 1944 to 1997 [40]. It was found that $H_{e}$ and $A$ were high and stable over time, but that $F_{\text {temporal }}$ varied substantially among periods. The authors concluded that high genetic diversity was maintained by gene flow in the face of small, local $N_{e}$, leading to the moderately high $F_{\text {temporal }}$.

$N_{e}$ monitoring usually is based on change in allele frequencies, and is similar to tests described above using $F_{\text {temporal }}$. However, although the above tests are sensitive to relative changes in population size, they do not quantify it. Estimating $N_{e}$ enables direct tests for changes in population size. As two samples are needed for a single temporal estimate of $N_{e}$, monitoring changes in $N_{e}$ via the temporal method requires samples from at least three time periods. $N_{e}$ has been estimated for brown bears in Yellowstone National Park by analyzing samples from the 1910s, $1960 \mathrm{~s}$ and $1990 \mathrm{~s}$ [41]. $N_{e}$ estimates were $\sim 85$ for both time periods (1910-1960s and 1960s-1990s), providing no evidence of a recent population decline. Other studies have also used a combination of contemporary and historical samples to obtain multiple temporal estimates of $N_{e}$ [42,43]. Palm et al. [44] monitored allele frequencies for 20 years in two Swedish brown trout populations and used a modified temporal method to obtain annual estimates of $N_{e}$. They found consistently small estimates, but no temporal trend in $N_{e}$.

$N_{e}$ monitoring can also be based on gametic disequilibrium, requiring only one sample for each $N_{e}$ estimate. Unbiased gametic disequilibrium $N_{e}$ estimators have been available only recently $[45,46]$. The precision and reliability of this method have not yet been thoroughly quantified, and further research is needed. However, the ability to estimate $N_{e}$ based on a single sample should improve the power to estimate any trends.

Gene flow Effective conservation often depends on the identification of management units and timely information regarding the effects of natural and anthropogenic factors on movement and gene flow between these units [47]. Although several genetic methods yield point estimates of gene flow, a monitoring program that produces a temporal series of samples can provide richer insights [48,49]. Furthermore, monitoring changes in gene flow indices, such as $F_{\mathrm{ST}}$, can detect changes in differentiation among populations [50]. For example, genetic monitoring of leopard frog Rana pipiens populations revealed that genetic structure was stable over 11-15 generations [51]. A study of cod Gadus morhua populations from the Baltic and North Sea using both historical and contemporary samples also found high temporal stability in $F_{\mathrm{ST}}$ over 47 and 89 years, respectively [35]. However, other studies have found the opposite result. Genetic monitoring of Scottish red deer Cervus elaphus demonstrated that fine-scale genetic structure in females declined at a steady rate over a 24-year period [52]. Based on demographic data collected over the same time period, the authors concluded that this decline was due to a combination of increasing population size and decreases in polygyny.

One of the most fruitful uses of genetic monitoring is likely to be the quantification of changes to movement patterns in response to events that disrupt historical patterns of connectivity, such as habitat fragmentation. Genetic monitoring is already a useful tool for evaluating the cumulative effects of habitat fragmentation. An allozyme and microsatellite-based study of California valley oak Quercus lobata pollen movement suggests that there was a decline in the effective number of fathers contributing pollen to the next generation between 1944 and 1999 [53]. The authors propose that this was the result of progressive stand thinning and that it might lead to future reproductive failure owing to genetic isolation. In a study of Pyne's ground plum Astragalus bibullatus, the genetic diversity of allozymes in multiple stratigraphic deposits 
was examined to discern the effects of cedar glade fragmentation [54]. Seeds from the top soil layer had higher levels of differentiation among sites than did the two lower layers, a result consistent with recent habitat loss.

Population mixtures Mixed-stock analysis estimates the proportions of individuals in a mixture that originate from each of two or more genetically differentiated breeding groups. Although mixed stock analysis (and detection of hybrids) can be based on diagnostic assays (Category Ib), it is often necessary to use probabilistic approaches based on allele or genotype frequencies. Genetic tools have been used for many years to assess the composition of mixedstock fisheries $[55,56]$, but recent laboratory and statistical advances provide increased power for real-time genetic monitoring. Fraser River sockeye salmon Oncorhynchus nerka are the most valuable commercial salmon fishery in British Columbia, but efficient harvest of the summer run is complicated by conservation concerns for late-returning populations. In 2002, a large monitoring program (up to 600 individuals several times a week for two months) based on microsatellites and major histocompatibility (MHC) loci provided stock composition estimates within 9-30 h [57], enabling managers to avoid overharvest of the late run.

Population admixtures also can be quantified and monitored with methods that estimate the degree of mixed ancestry of each individual. However, the power and resolution are generally much lower when the source populations are characterized by allele frequency differences, rather than by fixed differences in diagnostic markers ([56] as in Category Ib).

\section{Caveats to genetic monitoring}

Genetic monitoring offers some of the best opportunities to track populations over time and to evaluate when populations reach critical thresholds that demand management action. Category I genetic monitoring has many of the benefits of traditional abundance, distribution and vital rate monitoring, with the added benefit of larger and, in some cases, more representative samples owing to the relative ease of non-invasive genetic sampling. Category II monitoring measures genetic variation, which is the raw material of evolution and which affects the long-term status of a population. However, there are limitations to recognize and cautions to heed when implementing genetic monitoring programs.

First, DNA markers represent an added expense, particularly with non-invasive samples, owing to the need to repeat genetic analyses to reduce genotyping errors [58]. This expense is often offset by reductions in field costs, such as those incurred by the handling of live animals. Second, genotyping errors, if not adequately controlled, impede accurate estimates of many metrics. Multifold overestimates in abundance (Category I) can occur if genotyping error rates are high [59]; by contrast, $N_{e}$ estimated from the temporal method (Category II) often will be downwardly biased if genotyping errors are common, as errors will be interpreted as drift. Given the desire for retrospective monitoring using archived samples, which often yield low-quality DNA, genotyping error must be aggressively guarded against. Third, genetic monitoring efforts that rely on non-invasive samples are more vulnerable than are traditional monitoring to fraud (e.g. it is easier to plant a spurious genetic sample than a live individual); conflict has already arisen over planting of hair samples in a noninvasive survey designed to monitor the occurrence of a threatened species [23]. Fourth, sufficient molecular, statistical, and computational tools are not always available to detect the phenomenon of interest with the desired power (Box 3). For instance, in most cases, detection of hybridization is limited to $F_{1}$ and occasionally $F_{2}$ generations, largely owing to the lack of diagnostic markers [60]. Lastly, perhaps the main limitation is that genetic changes associated with population declines or fragmentation are most easily detected following severe disturbances. This limitation can be overcome to some extent by using more

\section{Box 3. Designing a genetic monitoring program}

Most regular monitoring efforts will yield useful information, even though the most valuable aspects might not be apparent until decades later. To maximize prospects of obtaining useful results, numerous factors should be considered in monitoring program design:

\section{Identify objectives}

Long-term monitoring programs might begin with rather general objectives that are likely to evolve over time. Shorter-term efforts generally have more focused objectives that should be as specific as possible. For example: (i) estimate abundance or $N_{e}$ of a target population with a coefficient of variation $\leq 0.5$; or (ii) have a $95 \%$ probability of detecting invasive species (or hybrids or immigrants) in the study area.

Alternatively, the primary objective might be to draw general conclusions about ecological impacts that will be applied to other populations (thus increasing the scope of inference); for example, the evaluation of whether dams alter migration patterns in an aquatic species over time.

\section{Evaluate potential sampling and analytical methods}

Potential sampling and analytical methods should be evaluated with respect to: (i) relevance to objectives. Can the method provide the right type of information? In general, long-term projects should begin with a relatively simple sampling design to provide flexibility for future changes; (ii) logistics. Is it feasible to collect the necessary data?; (iii) power. Given a feasible sampling regime, can the analytical method deliver the necessary statistical power to accomplish the objective?; and (iv) robustness. Unexpected events will occur; how sensitive are the methods to unforeseen developments and model assumptions?

\section{Include an experimental design}

Appropriate controls should be established whenever possible; if this is not feasible, changes in key population parameters can still be monitored, but it will be more difficult to establish cause-andeffect relationships. If the objectives involve broad inferences about ecological processes, a more complex experimental design is called for. For example, the traditional BACl (before-after, control-impact) design can be modified to enable more temporal and spatial replication of monitoring efforts [85].

\section{Manage adaptively}

The program should allow for maximum flexibility to respond to new information and unanticipated factors, including changes in the natural system (e.g. flood, drought or cyclic climate regimes); anthropogenic changes (uncontrolled modifications to system); and new analytical methods. If the program extends for any period of time, it is likely that important new methods will emerge. Can the data and sampling regime accommodate new methods? Can voucher specimens be archived for subsequent verification or to provide material for new (as yet unforeseen) analyses? 
and larger samples or more loci, which increases statistical power to detect effects of smaller size.

\section{Perspectives}

Although genetic monitoring has been discussed for some time [61], many techniques, statistics and models are relatively new. Consequently, limited time series exist to evaluate the true promise of genetic monitoring. Given the demonstrated power and flexibility of genetic monitoring, we believe that applications to answer key ecological, conservation and evolutionary questions will continue to expand rapidly.

Such developments are expected in the number and classes of molecular markers used for monitoring, demonstrated by the recent developments in population genomics and genome typing [4]. For instance, monitoring loci related to a stress event (e.g. MHC), along with many neutral markers for background information, could help detect the onset of selection events such as emerging diseases, anthropogenic landscape change, competition from invasive species, or pollution. Population genomics will also provide increased statistical power to monitor for genetic change. Advances will also occur in the monitoring of gene expression. For example monitoring changes in gene expression using microarrays [62] could signal a novel stressor (e.g. water quality change) or provide an early warning of detrimental stressors and challenges to organisms (e.g. emerging disease).

Other areas where progress is likely is in the development of sampling strategies, experimental designs (Box 3) and statistical approaches. New statistical frameworks such as approximate Bayesian computation, which are less computationally demanding, will be used more widely to estimate population parameters such as migration or $N_{e}$ $[4,6]$. Furthermore, a greater emphasis will probably be placed on estimating power to detect effects using the aforementioned metrics at a single point in time (e.g. power to detect substructure [63]) and power to detect trend in these metrics given the variability inherent in natural systems (Box 3; [64]). This will, in turn, force researchers to focus more on sampling strategies and experimental design and turn away from ad hoc study designs.

Finally, for Category II, we expect more monitoring of signals embedded in the genetic data, such as changes in microsatellite allele size and frequency distributions, and more monitoring of changes in individual-based gene flow models from the emerging field of landscape genetics (i.e. the use of assignment, and clustering algorithms to look at contemporary movement $[5,65])$. These new approaches have considerable promise for studying non-equilibrium systems, but the range of practical applications for which they can provide useful information has not yet been established [56].

\section{Future research}

We hope that our review will inspire additional research. First, simulation studies are needed to evaluate the relative power of monitoring genetic or traditional population metrics (e.g. to determine when it is more effective to monitor abundance or $N_{e}$ to detect changes of given magnitudes). Second, cross-fertilization of ideas between researchers studying different taxa is desirable, as we have noticed that monitoring with diagnostic molecular markers (Category Ia) is primarily used with terrestrial vertebrates, whereas monitoring population genetic parameters (Category II) is more common with other taxa. We see no biological imperative for this dichotomy. That cross-fertilization is already beginning to occur is illustrated by the use of non-invasive genetic monitoring to estimate abundance and harvest rates in coral reef fish [66].

Finally, we observe that much has been learned recently by analyzing historical samples that are only available through the foresight of previous collectors. These empirical results, together with the recognition that we cannot anticipate future technological advances, argue cogently for aggressive archiving contemporary samples. We never know what creative new approaches will be developed in the future for monitoring ecological change.

\section{Acknowledgements}

We thank Michael Hansen, Kevin McKelvey, Jeff Copeland, and two anonymous reviewers for helpful comments; John Boulanger for comments on Box 1; Luke Barrett for providing examples of genetic monitoring from the plant research community; and Stefan Himmer and John McMillan for their photographs of the Lake Owikeno brown bear and the Steelhead, respectively. Partial support to write this review was provided to M.K.S. through the Rocky Mountain Research Station and a PECASE award; G.L. was supported by the Luso-American Foundation (FLAD).

\section{References}

1 Holthausen, R. et al. (2005) Strategies for Monitoring Terrestrial Animals and Habitats, US Department of Agriculture, Forest Service, Rocky Mountain Research Station

2 Kurtz, J.C. et al. (2001) Strategies for evaluating indicators based on guidelines from the Environmental Protection Agency's Office of Research and Development. Ecol. Indic. 1, 49-60

3 United Nations Environment Programme Convention on Biological Diversity SBSTTA (2003) Monitoring and Indicators: Designing National-Level Monitoring Programmes and Indicators, United Nations

4 Luikart, G. et al. (2003) The power and promise of population genomics: from genotyping to genome typing. Nat. Rev. Genet. 4, 981-994

5 Manel, S. et al. (2003) Landscape genetics: the combination of landscape ecology and population genetics. Trends Ecol. Evol. 18, 189-197

6 Beaumont, M.A. and Rannala, B. (2004) The Bayesian revolution in genetics. Nat. Rev. Genet. 5, 251-261

7 Herbert, P.D.N. et al. (2004) Identification of birds through DNA barcodes. PLoS Biol. 2, 1657-1663

8 Baker, C.S. et al. (2000) Predicted decline of protected whales based on molecular genetic monitoring of Japanese and Korean markets. Proc. R. Soc. B 267, 1191-1199

9 White, G.C. (2005) Correcting wildlife counts using detection probabilities. Wildl. Res. 32, 211-216

10 Frantz, A.C. and Roper, T.J. (2006) Simulations to assess the performance of different rarefaction methods in estimating population size using small datasets. Conserv. Genet. 7, 315-318

11 Lukacs, P.M. and Burnham, K.P. (2005) Review of capture-recapture methods applicable to noninvasive genetic sampling. Mol. Ecol. 14, 3909-3919

12 Avise, J. (2004) Molecular Markers, Natural History and Evolution. Sinauer

13 Proctor, M.F. et al. (2005) Genetic analysis reveals demographic fragmentation of grizzly bears yielding vulnerably small populations. Proc. R. Soc. B 272, 2409-2416

14 Boulanger, J. et al. (2004) Monitoring of grizzly bear population trends and demography using DNA mark-recapture methods in the Owikeno Lake area of British Columbia. Can. J. Zool. 82, 12671277 
15 Piggott, M.P. et al. (2006) Estimating population size of endangered brush-tailed rock-wallaby (Petrogale penicillata) colonies using faecal DNA. Mol. Ecol. 15, 81-91

16 Prugh, L.R. et al. (2005) Monitoring coyote population dynamics by genotyping faeces. Mol. Ecol. 14, 1585-1596

17 Rudnick, J.A. et al. (2005) Using naturally shed feathers for individual identification, genetic parentage analysis, and population monitoring in an endangered Eastern imperial eagle (Aquila heliaca) population from Kazakhstan. Mol. Ecol. 14, 2959-2967

18 Flagstad, O. et al. (2004) Colonization history and noninvasive monitoring of a reestablished wolverine population. Conserv. Biol. $18,676-688$

19 Bailey, L.L. et al. (2004) Estimating site occupancy and species detection probability parameters for terrestrial salamanders. Ecol. Appl. 14, 692-702

20 Manley, P. et al. (2004) Evaluation of a multiple-species approach to monitoring species at the ecoregional scale. Ecol. Appl. 14, 296310

21 McKelvey, K.S. et al. (2006) DNA analysis of hair and scat collected along snow tracks to document the presence of Canada lynx (Lynx canadensis). Wildl. Soc. Bull. 34, 451-455

22 Fernandez, N. et al. (2006) Landscape evaluation in conservation: molecular sampling and habitat modeling for the Iberian lynx. Ecol. Appl. 16, 1037-1049

23 Mills, L.S. (2002) False samples are not the same as blind controls. Nature 415, 471

24 Schwartz, M.K. et al. (2004) Hybridization between bobcats and Canada lynx. Conserv. Genet. 6, 349-355

25 Miller, C.R. et al. (2003) Pedigree-based assignment tests for reversing coyote (Canis latrans) introgression into the wild red wolf (Canis rufus) population. Mol. Ecol. 12, 3287-3301

26 Haig, S.M. et al. (2004) Genetic identification of spotted owls, barred owls, and their hybrids: legal implications of hybrid identity. Conserv. Biol. 18, 1347-1357

27 Hitt, N.P. et al. (2003) Spread of hybridization between native westslope cutthroat trout, Oncorhynchus clarki lewisi, and nonnative rainbow trout, Oncorhynchus mykiss. Can. J. Fish. Aquat. Sci. 60, 1440-1451

28 Allendorf, F.W. et al. (2004) Intercrosses and the U.S. Endangered Species Act: should hybridized populations be included as westslope cutthroat trout? Conserv. Biol. 18, 1203-1213

29 Joly, D.O. and Messier, F. (2005) The effect of bovine tuberculosis and brucellosis on reproduction and survival of wood bison in Wood Buffalo National Park. J. Anim. Ecol. 74, 543-551

30 Briones, V. et al. (2000) Bovine tuberculosis and the endangered Iberian lynx. Emerg. Infect. Dis. 6, 189-191

31 Bricker, B.J. and Ewalt, D.R. (2005) Evaluation of the HOOF-Print assay for typing Brucella abortus strains isolated from cattle in the United States: results with four performance criteria. BMC Microbiol. 5,37

32 Kilpatrick, A.M. et al. (2006) West Nile Virus epidemics in North America are driven by shifts in mosquito feeding behavior. PLoS Biol. 4, 606-610

33 Barrett, L.G. et al. (2005) Temporal patterns of genetic variation across a 9-year-old aerial seed bank of the shrub Banksia hookeriana (Proteaceae). Mol. Ecol. 14, 4169-4179

34 Pertoldi, C. et al. (2005) Present and past microsatellite variation and assessment of genetic structure in Eurasian badger (Meles meles) in Denmark. J. Zool. 265, 387-394

35 Poulsen, N.A. et al. (2006) Long-term stability and effective population size in North Sea and Baltic Sea cod (Gadus morhua). Mol. Ecol. 15, 321-331

36 Leberg, P.L. (1992) Effects of a population bottleneck on genetic variation as measured by allozyme electrophoresis. Evolution 46, 477-494

37 Spencer, C.C. et al. (2000) Experimental evaluation of the usefulness of microsatellite DNA for detecting demographic bottlenecks. Mol. Ecol. 9, 1517-1528

38 Luikart, G. et al. (1999) Temporal changes in allele frequencies provide estimates of population bottleneck size. Conserv. Biol. 13, $523-530$

39 Ramakrishnan, U. et al. (2005) Detecting past population bottlenecks using temporal genetic data. Mol. Ecol. 14, 2915-2922
40 Ostergaard, S. et al. (2003) Long-term temporal changes of genetic composition in brown trout (Salmo Trutta L.) populations inhabiting an unstable environment. Mol. Ecol. 12, 3123-3135

41 Miller, C.R. and Waits, L.P. (2003) The history of effective population size and genetic diversity in the Yellowstone grizzly (Ursus arctos): implications for conservation. Proc. Natl. Acad. Sci. U. S. A. 100, 43344339

42 Hansen, M.M. et al. (2006) Underwater but not out of sight: genetic monitoring of effective population size in the endangered North Sea houting (Coregonus oxyrhynchus). Can. J. Fish. Aquat. Sci. 63, 780-787

43 Ardren, W.R. and Kapuscinski, A.R. (2003) Demographic and genetic estimates of effective population size $\left(N_{e}\right)$ reveals genetic compensation in steelhead trout. Mol. Ecol. 12, 35-49

44 Palm, S. et al. (2003) Effective population size and temporal genetic change in stream resident brown trout (Salmo trutta, L.). Conserv. Genet. 4, 249-264

45 England, P.R. et al. (2006) Estimating effective population size from linkage disequilibrium: severe bias in small sizes. Conserv. Genet. 7, 303-308

46 Waples, R.S. (2006) A bias correction for estimates of effective population size based on linkage disequilibrium at unlinked gene loci. Conserv. Genet. 7, 167-184

47 Palsboll, P.J. et al. Identification of management units using population genetic data. Trends Ecol. Evol. 22. (in press)

48 Koskinen, M.T. et al. (2002) Genetic assessment of spatiotemporal evolutionary relationships and stocking effects in grayling (Thymallus thymallus, Salmonidae). Ecol. Lett. 5, 193-205

49 Coulon, A. et al. (2006) Genetic structure is influenced by landscape features: empirical evidence from a roe deer population. Mol. Ecol. 15, 1669-1679

50 Ryman, N. et al. (1995) Introgression, supportive breeding, and genetic conservation. In Population Management for Survival and Recovery (Ballou, J.D. et al., eds), pp. 341-365, Columbia University Press

51 Hoffman, E.A. and Blouin, M.S. (2004) Historical data refute recent range contraction as a cause of low genetic diversity in isolated frog populations. Mol. Ecol. 13, 271-276

52 Nussey, D.H. et al. (2005) Rapidly declining fine-scale spatial genetic structure in female red deer. Mol. Ecol. 14, 3395-3405

53 Sork, V.L. et al. (2002) Pollen movement in declining populations of California Valley oak, Quercus lobata: where have all the fathers gone? Mol. Ecol. 11, 1657-1668

54 Morris, A.B. et al. (2002) Stratified analysis of the soil seed bank in the cedar glade endemic Astragalus bibullatus: evidence for historica changes in genetic structure. Am. J. Bot. 89, 29-36

55 Shaklee, J.B. et al. (1999) Managing fisheries using genetic data: case studies from four species of Pacific salmon. Fish. Res. 43, 45-78

56 Manel, S. et al. (2005) Assignment methods: matching biological questions with appropriate techniques. Trends Ecol. Evol. 20, 136142

57 Beacham, T.D. et al. (2004) DNA in action: rapid application of DNA variation to sockeye salmon fisheries management. Conserv. Genet. 5, $411-416$

58 Bonin, A. et al. (2004) How to track and assess genotyping errors in population genetic studies. Mol. Ecol. 13, 3261-3273

59 Waits, J.L. and Leberg, P.L. (2000) Biases associated with population estimation using molecular tagging. Anim. Conserv. 3, 191-199

60 Vaha, J.P. and Primmer, C.R. (2006) Efficiency of model-based Bayesian methods for detecting hybrid individuals under different hybridization scenarios with different numbers of loci. Mol. Ecol. 15, 63-72

61 Allendorf, F.W. and Ryman, N. (1987) Genetic management of hatchery stocks. In Population Genetics and Fishery Management (Ryman, N. et al., eds), pp. 141-159, University of Washington Press

62 Whitehead, A. and Crawford, D.L. (2006) Variation within and among species in gene expression: raw material for evolution. Mol. Ecol. 15, $1197-1211$

63 Ryman, N. et al. (2006) Power for detecting genetic divergence: differences between statistical methods and marker loci. Mol. Ecol. $15,2031-2045$

64 Gerrodette, T. (1987) A power analysis for detecting trends. Ecology 68, 1364-1372

65 Cushman, S.A. et al. Gene-flow in complex landscapes: testing multiple hypotheses with causal modeling. Am. Nat. (in press) 
66 Hoyle, S.D. et al. (2005) LOCUSEATER and SHADOWBOXER: programs to optimize experimental design and multiplexing strategies for genetic mark-recapture. Mol. Ecol. Notes 5, 974976

67 Waples, R.S. (1989) A generalized approach for estimating effective population size from temporal changes in allele frequency. Genetics 121, 379-391

68 Wang, J. (2001) Optimal marker-assisted selection to increase the effective size of small populations. Genetics 154, 475-489

69 Tallmon, D.A. et al. (2004) Comparative evaluation of a new effective population size estimator based on approximate Bayesian computation. Genetics 167, 977-988

70 Forney, K.A. (2000) Environmental models of cetacean abundance: reducing uncertainty in population trends. Conserv. Biol. 14, 12711286

71 Boulanger, J. and McLellan, B. (2001) Closure violation in DNA-based mark-recapture estimation of grizzly bear populations. Can. J. Zool. $79,642-651$

72 Paetkau, D. (2003) An empirical exploration of data quality in DNAbased population inventories. Mol. Ecol. 12, 1375-1387

73 Pradel, R. (1996) Utilization of mark-recapture for the study of recruitment and population growth rate. Biometrics 52, 703709

74 Waples, R.S. and Drake, J. (2004) Risk-benefit considerations for marine stock enhancement: a Pacific salmon perspective. In Stock Enhancement and Sea Ranching: Developments, Pitfalls and Opportunities (Leber, K.M. et al., eds), pp. 260-306, Blackwell

75 Dowling, T.E. et al. (2005) Genetic monitoring of wild and repatriated populations of endangered razorback sucker (Xyrauchen texanus, Catostomidae, Teleostei) in Lake Mohave, Arizona-Nevada. Mol. Ecol. 14, 123-135
76 Osborne, M.J. et al. (2006) Genetic effects of hatchery propagation and rearing in the Endangered Rio Grande silvery minnow, Hybognathus amarus. Rev. Fish. Sci. 14, 127-138

77 Jorstad, K.E. (2004) Genetic studies in marine stock enhancement in Norway. In Stock Enhancement and Sea Ranching Developments, Pitfalls, and Opportunities (Leber, K.M. et al., eds), pp. 339-352, Blackwell

78 Hansen, M.M. (2002) Estimating the long-term effects of stocking domesticated trout into wild brown trout (Salmo trutta) populations: an approach using microsatellite DNA analysis of historical and contemporary samples. Mol. Ecol. 11, 1003-1015

79 Araki, H. et al. Reproductive success of captive-bred steelhead trout in the wild: evaluation of three hatchery programs in the Hood River. Conserv. Biol. (in press)

80 Hedrick, P.W. et al. (2000) The impact of supplementation in winterrun Chinook salmon on effective population size. Genetics 91, 112-116

81 Ramp, J.M. et al. Restoration genetics of the vernal pool endemic Lasthenia conjugens (Asteraceae). Conserv. Genet. (in press)

82 Sigg, D.P. (2006) Reduced genetic diversity and significant genetic differentiation after translocation: comparison of the remnant and translocated populations of bridled nailtail wallabies (Onychogalea fraenata). Conserv. Genet. 7, 577-589

83 Milbury, C.A. et al. (2004) Mitochondrial DNA markers allow monitoring of oyster stock enhancement in the Chesapeake Bay. Mar. Biol. 2004, 351-359

84 Selosse, M-A. et al. (1998) Temporal persistence and spatial distribution of an American inoculant strain of the ectomycorrhizal basidiomycete Laccaria bicolor in a French forest plantation. Mol. Ecol. $7,561-573$

85 Underwood, A.J. (1994) On beyond BACI: sampling designs that might reliably detect environmental disturbances. Ecol. Appl. 4, 3-15

\section{Elsevier.com - linking scientists to new research and thinking}

Designed for scientists' information needs, Elsevier.com is powered by the latest technology with customer-focused navigation and an intuitive architecture for an improved user experience and greater productivity.

The easy-to-use navigational tools and structure connect scientists with vital information - all from one entry point. Users can perform rapid and precise searches with our advanced search functionality, using the FAST technology of Scirus.com, the free science search engine. Users can define their searches by any number of criteria to pinpoint information and resources. Search by a specific author or editor, book publication date, subject area - life sciences, health sciences, physical sciences and social sciences - or by product type. Elsevier's portfolio includes more than 1800 Elsevier journals, 2200 new books every year and a range of innovative electronic products. In addition, tailored content for authors, editors and librarians provides timely news and updates on new products and services.

Elsevier is proud to be a partner with the scientific and medical community. Find out more about our mission and values at Elsevier.com. Discover how we support the scientific, technical and medical communities worldwide through partnerships with libraries and other publishers, and grant awards from The Elsevier Foundation.

As a world-leading publisher of scientific, technical and health information, Elsevier is dedicated to linking researchers and professionals to the best thinking in their fields. We offer the widest and deepest coverage in a range of media types to enhance cross-pollination of information, breakthroughs in research and discovery, and the sharing and preservation of knowledge.

\section{Elsevier. Building insights. Breaking boundaries. www.elsevier.com}

